\title{
Using ethnographic methods to carry out human factors research in software engineering
}

\author{
J. S. KARN and A. J. COWLING \\ University of Sheffield, Sheffield, England
}

\begin{abstract}
This article describes how ethnographic methods were used to observe and analyze student teams working on software engineering (SE) projects. The aim of this research was to uncover the effects of the interplay of different personality types, as measured by a test based on the Myers-Briggs Type Indicator (MBTI), on the workings of an SE team. Using ethnographic methods allowed the researchers to record the effects of personality type on behavior toward teammates and how this related to the amount of disruption and positive ideas brought forward from each member; also examined in detail were issues that were either dogged by disruption or that did not have sufficient discussion devoted to them and the impact that they had on the outcomes of the project. Initial findings indicate that ethnographic methods are a valuable weapon to have in one's arsenal when carrying out research into human factors of SE.
\end{abstract}

The goal of this article is to describe how ethnographic methods were used to study software engineering (SE) teams for the duration of specific projects, in order to gain a greater understanding of the role of human factors over the duration of an industrial SE project. Group interaction styles affect communication and, thus, team performance by facilitating or hindering the exchange of information among group members. An issue requiring further study was how constellations of personality types are manifested in team interaction styles.

Taking this into account, the work described here also had another dimension, which was to study how particular combinations of individual personalities, as measured by an online version of the Myers-Briggs Type Indicator (MBTI), might either promote effective teamwork or cause clashes and, second, how these clashes might disrupt the operations of an SE team.

The personal interest behind this research lies in teams and the psychological aspects of SE. Most SE projects are carried out by teams of varying size, working mythical man months (Brooks, 1975), in often feverish attempts to meet project deadlines. Teams outperform individuals acting alone or in larger organizational groupings, especially when performance requires multiple skills, judgments, and experiences. A team is more than just several people working on a common project, although the level of teamwork will vary from one team to another. Teamwork represents a set of values that encourage such behaviors as listening and constructively responding to points of view expressed by others, giving others the benefit of the doubt, providing support to those who need it, and recognizing

Correspondence should be addressed to J. S. Karn, Department of Computer Science, University of Sheffield, Sheffield S1 4DP, England (e-mail: j.karn@sheffield.ac.uk). the interests and achievements of others. The importance of teamwork in SE has been recognized at a pedagogic level. Many undergraduate courses now have group projects as a central part of their syllabus. These projects can vary from carrying out the theoretical analysis and design of a system to the analysis, design, and implementation of a system for an external industrial client.

A computer-based system is built for people and by people, it is intended to serve some human purpose, and it exists in some human context. Some of the most vexing difficulties involved in SE projects are social, political, or cultural, and social issues are inherent to the requirementsengineering process. The work carried out by the present authors was not limited to understanding requirements engineering but was intended to encompass the entire SE project lifecycle.

So far, the authors have studied the operation of teams throughout complete projects, measuring the levels of disruption that different issues have caused to the team, and have shown how these disruptions can be related to personality clashes between individuals within the teams (Karn \& Cowling, 2004a, 2004b). Although there are numerous advantages to working in teams, there are also several problems that could pose a potential risk to SE teams, since a team approach to a project usually generates a greater degree of conflict than many people are accustomed to. Many disruptions were observed, ranging from sarcasm in response to points raised to angry screaming matches. Personality clashes, nonparticipants, and one group member's doing all of the work can be very serious problems and are often detrimental to a team's performance. If such problems are not dealt with early on in the process, there is a real danger that the team will become totally dysfunctional.

In follow-up work (Karn \& Cowling, 2004c), we have examined the effects of disruptions and, in particular, 
how they have affected both the cohesiveness of the team and the quality of the work produced. The initial expectations were that a team that experienced a large amount of disruption would encounter the most serious amount of destruction in terms of the quality of the work produced and that such disintegration would have cataclysmic repercussions on the kinship and rapport between different team members. This was what had been observed in earlier studies (Karn \& Cowling, 2004a, 2004b) and was thought to be the most likely source of discontent at the outset of the work described in this report. At the start of the project, little attention was paid to the notion that failure to debate issues effectively could have a negative impact on the workings of a team throughout the project. What was found was that this proved to be just as serious a problem as serious disruptions.

In terms of looking at the interplay between different personalities, what are needed are measurements and qualitative descriptions of how and why certain teams perform better than others and the effect that this has on the work produced.

Therefore, to get an accurate picture of how well a team has performed in a project, it is necessary to utilize ethnographic methods to observe and measure the team throughout the duration of the project; only then is a researcher in the position to state that a particular team did work well throughout the project. The long-term realistic goal of this research is to identify those aspects of personality that may be particularly significant and to give deep qualitative descriptions of them.

The context for this study was the Software Engineering Observatory at the University of Sheffield, a research facility that is run by the Verification and Testing (VT) research group. This is an attempt by the University of Sheffield to create a professional SE environment within the university (Cowling, 1994, 1997). Researchers from the VT group make extensive use of the observatory when carrying out empirical work. The observatory allows empirical researchers to observe, question, or interview software developers working on real industrial projects. A whole range of SE group projects take place each year in the Department of Computer Science. These include the Crossover, Software Hut, Genesys, Darwin, and Maxi Projects. These projects vary from first-year undergraduate courses to MSc projects.

The remainder of this article will describe the methodology used to ascertain the aforementioned results, with a focus on explaining the evolution of the research strategy.

\section{Personality Typology Used}

The personality theory underlying this research was provided by Jung's (1923) work on psychological types, as developed by Myers and Briggs in designing the Myers-Briggs Type Indicator (MBTI; Myers \& Myers, 1987). The test used in this research is not the official paper version of the MBTI, but an online test based on the MBTI developed by Human Metrics, a consortium of Israeli psychologists, who claim that they have found no significant statistical differences between this test and the official version of the MBTI.

The MBTI is used to assess an individual's personality style on four dimensions: social interaction, information gathering, decision making, and dealing with the external world. It is a tool that can be grasped and understood in a relatively short time to give a better understanding of people and their differences.

A brief overview of MBTI functions and attitudes is necessary at this point.

Sensing. The sensing type depends on the five senses for perception. Whatever comes directly from the senses is part of the sensing type's own experience and is, therefore, trustworthy. Anyone preferring sensing to intuition is primarily interested in actualities.

Intuition. The intuitive type listens for the intuitions that come up from the unconscious with enticing visions of possibilities. Intuitives are mainly interested in possibilities. The only fields that interest them are those that give inspiration some play. They abhor the routine, since it leaves nothing for inspiration to accomplish.

Thinking. Thinking is essentially impersonal. Its goal is objective truth, independent of the personality and wishes of the thinker or anyone else. So long as the problems are impersonal and can be judged from the true-false standpoint, thinking is the better instrument.

Feeling. Human motives are notably personal. Therefore, in the sympathetic handling of people, where personal values are important, feeling is the most effective instrument.

Judging. When using this attitude to come to a conclusion, people will shut off perception for the time being. All the evidence is in, and anything else is irrelevant; the time has come to arrive at a verdict.

Perceiving. In this attitude, people shut off judgment. Not all of the evidence is in; new developments will occur. It is much too soon to do anything irrevocable.

According to this theory, there are two distinct and sharply contrasting ways of perceiving. One means is through the process of sensing, by which one becomes aware of things directly through the five senses. The other is the process of intuition, which is indirect perception by way of the unconscious, incorporating ideas or associations that the unconscious tacks onto perceptions coming from outside. When people prefer sensing, they are so interested in present actualities that they pay little attention to ideas and insights that could come from nowhere, whereas a person who prefers intuition will be so engrossed in pursuing future possibilities that he or she will neglect and even fail to look at current actualities. There are also two distinct ways of judging. One way is the use of thinking; this is a logical process aimed at an impersonal finding. The other is by feeling; this relies on appreciation and bestows a personal, subjective value on things. The thinking/feeling preference is entirely independent of the sensing/intuition preference. Either kind of judgment can team up with either kind of perception.

The official MBTI is a Jungian-based inventory that uses a pen-and-paper self-report format. It is composed 
of 94 forced choice items that constitute the four bipolar discontinuous scales that are implied in Jung's theory: introversion-extraversion (I-E), sensation-intuition $(\mathrm{S}-\mathrm{N})$, thinking-feeling $(\mathrm{T}-\mathrm{F})$, and judging-perceiving (J-P). Respondents are classified into one of 16 personality types on the basis of the largest score obtained for each bipolar scale (i.e., a person scoring higher on extraversion than on introversion, on intuition than on sensing, on thinking than on feeling, and on judging than on perceiving would be classified as an extraverted, intuitive, thinking, judging type). On completion of the test, a subject will also know the clarity of preference for each function and attitude. An example is the following: I, 52\%; N, 33\%; $\mathrm{T}, 22 \% ; \mathrm{J}, 42 \%$. An indicator for clarity of preference is the following: $40 \%$ or higher $(30 \%$ for $\mathrm{T} / \mathrm{F})$, very clear preference; $31 \%-39 \%(21 \%-29 \%$ for $\mathrm{T} / \mathrm{F})$, clear preference; $11 \%-20 \%$, moderate preference; $1 \%-10 \%$, slight preference.

Neither the official nor the human metrics typology explains whether a person is psychologically healthy or unhealthy. When discussing or thinking about the use of psychological tests, one must always keep in mind possible negative effects on the research subjects. Research ethics should always be at the forefront of any researcher's mind when dealing with human subjects. This is another major advantage in favor of this typology. All of the categories are neutral. No type or preference is better than any other. This typology does not explain whether a person is healthy or unhealthy. It does not explain IQ, maturity, emotions, or many other things. This is a major plus point for any researcher, who can rest assured that on completion of the test, students will not be told that they have a propensity toward a potentially volatile label, such as schizoid, sociopath, hysteric, neurotic, or psychopath.

Another popular personality test is the NEO-PI model, which is based on the five-factor model of trait personality (Costa \& McCrae, 1992). The NEO-PI is a paperbased model, and no online version was found. Therefore, persuading students to take the test and return the results would have been more problematic and time consuming, given that the work took place according to a strict project timetable; students would have been less likely to volunteer to take part in the research.

\section{Research Methodology}

Ethnographic methods were employed to observe selected student teams. In order to gain an in-depth understanding of the user culture (in this context, SE teams), the authors observed and inquired about the research subjects' normal activities throughout their specific projects.

Ethnography is a rigorous research method in which good data collection and analytical skills are vital. The aim of this method is to allow the researcher to arrive at a theoretically comprehensive understanding of a group or culture. One of the key strengths of this method is that it allows one to gain an "inside" account of the lives of people under observation. Ethnographic research methods were originally founded by social anthropologists to aid them in their understanding of different cultures and environments. They are now used in many disciplines in which research involving humans is important, including empirical software engineering (ESE). To the social anthropologists, ethnography was synonymous with living in and immersing oneself in the culture of the group to be researched for an extended time period.

To those who are prepared and willing, it is a rewarding method that yields fascinating insights into people's social lives and relationships and, more generally, assists in bridging the gap between people's understanding of alternative lifestyles and the actual reality. Ethnographic researchers usually work alone; they witness the reflexive rationalization of conduct that is the continual interpretation and application of new knowledge by people in their social environments as an ongoing process. Ethnographers gather data by their active participation in the social world; they enter a social universe in which people are already busy interpreting and understanding their environments.

Practitioners shun what is known as the a priori (a proposition that can be known to be true or false without reference to experience), preferring the a posteriori (knowing how things are by reference to how things have been or are). Ethnographers believe that it is important to participate in social relations and seek to understand actions within the context of an observed setting. Why? Because it is argued that people act and make sense of their world by taking meanings from the environment. This technique, it is argued, is least likely to lead to researchers' imposing their own reality on the social world that they seek to understand. These strands of thought also combine with the idea of naturalism, which proposes that as far as possible, the social world should be studied in its "natural state," undisturbed by the researcher.

Ethnographers claim that the process of learning behavior is absent from other forms of research. For instance, a questionnaire asks questions at a particular time. It is a static, causal snapshot of attitudes; how and why people change is not understood. The design of a questionnaire involves the researchers' developing ideas and testing or exploring them, using questions. Critics argue that researchers using this method already know what is important. In contrast, ethnography is said to make no firm assumptions about what is important. Instead, the method encourages researchers to immerse themselves in the dayto-day activities of the people whom they are attempting to understand. In contrast to ideas being tested (deductive), they may be developed from observations (inductive). Ethnography leads to an empathic understanding of a social scene. It is said to exclude, over time, the preconceptions that researchers may have and to expose them to a new social milieu that demands their engagement.

Some of the key positive aspects of ethnographic methods can be summarized as the following. (1) They are least likely to lead researchers to impose their own reality on the social world they seek to understand. (2) The process of understanding action is omitted from other forms of research, and how and why people change is not understood. (3) During interviews, there may be language or cultural differences expressed; in this case, observers may record 
their own experiences in order to understand the cultural universe that people occupy (subjective experiences) and to convey these observations to a wider audience (from field notes), within the context of explaining the data (theoretical framework).

To become part of a social scene and participate in it requires that the researcher be accepted to some degree. This permits researchers to witness people's actions in different settings and routinely ask themselves a myriad of questions concerning motivations, beliefs, and actions. Examples of such questions are the following. Why did that happen and to whom? What do people ordinarily do in this setting and why? What would happen if people did $\mathrm{X}$ or $\mathrm{Y}$ ? What are the usual roles of the social scene? How are the rules negotiated? What are the verbal and nonverbal gestures employed? Who said what, to whom, and why? What do they mean, and how do they relate to particular relationships and actions?

Whyte (1984) used what he termed an orienting theory, which simply tells us, in the most general terms, what data we are likely to need at the point of analysis. Data are collected under two headings: (1) the identification of relationships within the social setting, and (2) the description of events and situations that took place.

Observation and the writing up of notes under these headings, together with any relevant interview data, provide a rich insight into social relations, events, and processes. Data collection and analysis and the decision as to when to withdraw from fieldwork then take place according to what is referred to as theoretical sampling and theoretical saturation, the latter referring to the time when observations no longer serve to call into question or modify the theories generated from earlier observations, thus rendering the theory saturated with data.

Atkinson (1990) stated that the final result of an ethnographic study is a text that attempts to persuade the audience of the authenticity of one's descriptions and their analyses. In a sense, writers have focused on texts not only as reporting a reality "out there," but also in terms of their abilities to construct social reality.

Application of ethnographic methods. Five teams were observed in the study, but due to space constraints, only one will be described in detail in this article. Full details for all the teams are given in Karn and Cowling (2004c). There were several phases involved in going from the field note stage to quantifiable data in the research described in this article; this theoretical sampling is described in detail in Karn and Cowling (2004a, 2004b). A pilot study was conducted over the course of a semester; during this study, field notes were taken during each team meeting. All the contributions from each member were noted, as was any interaction with the client or manager. After giving the field notes some kind of order, the next step was to classify aspects of the ordered details, such as identifying the kind of issue to which an idea, question, or problem was related. Since many meetings were attended, a large amount of data was gathered over the course of the study. During the projects, the focus was on gathering, as opposed to analyzing, data; at this stage, the authors had no clear ideas about how to classify the bewildering conglomeration of issues that were being discussed during the meetings. The MBTI results for the relevant team are given in Table 1.

On the whole, this team worked well, but sometimes, there was almost too much emphasis on not offending people. This meant that work was sometimes not done and there were no recriminations. In some cases, more discipline was needed, as well as a clear plan.

Genesys Team 4 had a majority of feeling types. They tended to weigh how deeply they cared about things that could be gained or lost by each of the alternatives available when making a decision. The prominent members of the group ensured that each final decision had a sound basis, because they took into account facts, possibilities, and human values. They also had a majority of intuitives, who were willing to accept ideas if they sounded reasonable and would help develop possibilities.

One member of this team (4F) was an ENTJ, with very clear preferences for the chosen functions and attitudes. 4F's no-nonsense, efficient, and often boisterous approach to decision making did not go down too well with certain introverted feeling types and proved to be a major source of animosity and conflict. Potentially explosive situations were usually defused by excellent negotiation and people management skills from senior members of the team, especially $4 \mathrm{~B}$.

Analysis of the field notes on completion of the observations revealed several prominent themes; these themes became the headings for a nominal scale that was created to classify issues: PSI, project-specific issue; MSI, methodology-specific issue; GSEI, general software engineering issue; THF, team human factors; C, client; and $\mathrm{M}$, manager.

The adaptation of the classification scheme described above was based on personal experiences, observations, and mental models of the events that take place during a typical group meeting; regardless of the scenario, the observations were closely related to the work of Becker (1993), who listed four distinct stages of analysis, whose overall aim was the categorization of collected data in order that the events, relationships, and interactions observed may be understood or explained within the context of a developed theoretical framework.

Since this was an ESE project, the meetings were related to SE. This did not affect the original ideas behind the classification; it simply meant that it had to be extended

Table 1 MBTI Results for Genesys Team 4 (in Percentages)

\begin{tabular}{llllll}
\hline ID & Type & E-I & S-N & T-F & J-P \\
\hline 4A & ISFJ & I34 & S44 & F24 & J22 \\
4B & ENFJ & E22 & N78 & F11 & J33 \\
4C & INTJ & I67 & N11 & T22 & J33 \\
4D & INFP & I44 & N22 & F28 & P36 \\
4E & INTJ & I33 & N22 & T44 & J44 \\
4F & ENTJ & E88 & N67 & T74 & J56 \\
\hline
\end{tabular}

Note-E, extroversion; I, introversion; $S$, sensing; N, intuition; T, thinking; F, feeling; J, judging; P, perceiving. 
to include specialist SE sections. Having some experience from participation and observations in meetings provided the framework that drove and underpinned the development of the classification system used in this project.

The process of analyzing the field notes and classifying data under the headings above was a long, arduous process, but the end result was that the authors gained a rich insight into social relations, events, and processes within SE teams. The derivation of ordinal scales to measure the impact of issues and the levels of disruption that they caused arose from the analysis of the fieldwork. Each issue was traced from its inception to its consummation, and this involved analyzing the relevant field notes in order to classify each specific issue.

An ordinal scale, called the level of disruption, was created to classify each issue and is shown in Table 2. This ordinal scale would give order to a rough grouping of numerical values developed earlier in the project. This scale was based on categories that arose from observing the teams.

Thus, the level of disruption ordinal scale consists of two groups of three. The first three ratings signify that an agreement was reached or an issue was resolved immediately, after a brief debate, or after an extended constructive debate. In essence, the ratings in this first group point to a lack of disruption and to the success of harmonious teamwork, although a large number of issues rated 1 could also indicate a problem, since it would show a lack of communication and interaction between the team members. In sharp contrast, the second group of ratings correspond to varying degrees of disruption, ranging from forcing people off relevant issues via sarcasm, pedantry, or anger to screaming matches that left people trembling with anger and issuing threats of violence.

After these scales had been completed, the information was recorded in a database. It could then be analyzed to determine, for instance, how many issues of each type or of each ordinal rating had occurred, or how many had involved particular individuals or combinations of individuals. The results of these analyses could be presented in bar charts, showing, for instance, which kinds of issues were dealt with smoothly, which caused disruption, and which involved some kind of personality conflict.

The levels of disruption for all the issues involving Genesys Team 4 are given in Figure 1.

The ordinal scales described in Karn and Cowling (2004a, 2004b) add to the reliability of the research car- ried out. Essentially, they allow any researcher to carry out an analysis of field notes and then to make an objective measurement of each issue.

Identifying and measuring issues and recording relevant information was a time-consuming and complex exercise. In later projects, it became easier to record information and to identify specific issues during the analysis of data. All of the displayed behavior from the research subjects was viewed through the perspective of their personality type; therefore, data recorded in the field notes had to be backed up by congruous information relating to the personality type of the relevant individual(s). This was another convoluted exercise, since the actions of one person with a particular personality type had to be balanced against their teammates' personality types; this helped the authors to identify personality clashes and how they disrupted the workings of the team. The final aspect of this process was that of anonymizing the results, and this was done in two stages. Each person was given a randomly generated number within each team, such as A1 and B2, to ensure that complete anonymity was achieved.

$4 \mathrm{~B}$ and, to a lesser extent, $4 \mathrm{~A}$ were the driving forces behind this team. These two members complemented each other and had an excellent working relationship. 4B is an ENFJ, and 4A is an ISFJ. 4B worked hard to make the other team members feel at ease under all circumstances; he was able to adapt individuals to the objective situation. 4B acted as the unofficial manager of the team and aimed to form and maintain harmonious relationships with other members of the team; 4B expressed himself eloquently and easily and made good efforts to establish warm sympathy and understanding between all the members.

Genesys Team 4 had several client-related problems, especially with regard to requirements. There was a lot of debate about the length of reports, which carried on into the later stages of the project. The documentation served to confuse the team, since to them, it seemed to be going against the tenets of Extreme Programming (XP), which emphasizes agility and tries to move away from a large amount of requirement documentation. People were still confused about documentation very late in the project. There was a suggestion from $4 \mathrm{~A}$ that the team should use a formal document to supplement the XP story cards; this would create extra documentation. The team went along with this idea but encountered more problems when the client modified the requirements document; this forced the team to complain that it had no structure. As a result

Table 2

Level of Disruption Ordinal Scale

\begin{tabular}{cl}
\hline Ordinal ID & \multicolumn{1}{c}{ Ordinal Name } \\
\hline 1 & Premise uncritically accepted, with no interaction between team members \\
2 & Dealt with smoothly and harmoniously after brief discussion \\
3 & Lengthy period of constructive debate discussing the virtues of an issue \\
4 & Caused slight disruption by forcing people off relevant issues \\
5 & Lengthy period of destructive debate, meeting disrupted, a lot of time \\
& wasted getting back on track \\
6 & Caused complete disruption to the work of the team \\
\hline
\end{tabular}




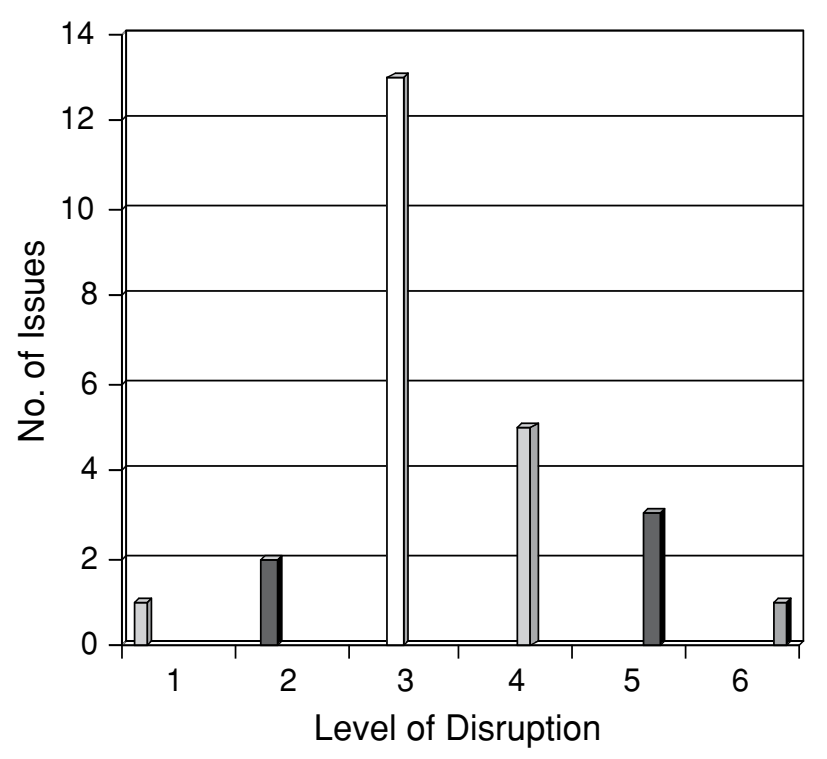

Figure 1. Level of disruption for all the issues for Genesys Team 4.

of this, the team had to rewrite its own and the client's requirements document. Every month, a Genesys team has to produce a monthly report; for Team 4 , many of these reports were rushed and lacked structure. Another problem was that they never managed to document all of the requirements from the first stage of the project; therefore, Stage 1 requirements were incomplete, and marks were lost. $4 \mathrm{~F}$ allowed his anger to boil over on one occasion and issued a threat of violence against the client - not to his face, but in the company of teammates. 4F's temper was a problem on several occasions and strained relations with the client and other team members.

Additional ordinal scales. Issues rated 1, 4, 5, and 6 on the level of disruption ordinal scale were deemed to be potentially problematic, for a variety of reasons. Issues dealing with disruption range from stifling debate with sarcastic or sharp comments to full-blown screaming matches and threats of violence. Issues that rated 1 on the scale could also be very damaging to a team, even in the absence of any disruption. Some degree of conflict among teammates is indicative of the introduction of a variety of ideas. This is seen as a positive function of group projects, providing that there is an established mechanism for conflict resolution and decision making. Conflict resolution can occur by compromise, forcing, avoidance, or confrontation.

Taking into account each issue that was dogged by either disruption or inadequate debate, the following ordinal scales were created: one dealing with issues that were resolved without management intervention, therefore causing an internal impact (see Table 3), and the other with issues resolved when management was forced to intervene due to the seriousness of the situation, resulting in an external impact (see Table 4). The aim of the scales is to highlight the level of destructiveness caused by a par- ticular disruption or lack of debate concerning a specific issue.

The ordinal ratings for the internal and external impacts for the observed team are given in Figures 2 and 3.

The ordinal ratings (internal and external impacts) related to the effect of a particular disruption or lack of debate and allowed one to see whether or not such a problem had any serious impact on a single part or the whole of the project. The scale showing the level of chaos without any management involvement ranged from no impact to no working system at the end of the project. The scale showing how much damage was inflicted if the manager was involved ranged from comments/concern to marks lost for an area of the project. The additional ordinal scales proved to be vital, since they allowed the authors to ascertain the full impact of the failure to discuss a topic or how much real damage was caused by an altercation between team members.

The ordinal scales give another dimension to the research. In addition to pointing out disruptive points on an MBTI map, the ratings allow one to numerically quantify all the issues that have arisen within a particular team, thereby creating a detailed profile of overall team and individual behavior throughout a project. Great effort went into devising the ordinal scales used throughout this research. The rationale behind this was to create scales that were sufficiently well defined that anyone else working in this area could conceivably make use of them.

Several patterns arose that were common to several teams across several projects. They were divided into three separate areas: user centered, technical, and sociopolitical.

User-centered problems typically refer to client-related issues; examples are meeting the client and additional/ changed requirements. Technical problems relate to the actual analysis, design, and implementation of the software system. They were divided into methodology related, database related, interface/Web site design, and documentation/specifications. Sociopolitical issues referred to team or human problems, such as apathy or poor communication.

Several user-centered problems were noted while Genesys Team 4 was observed. Having meetings and listening to ideas from the client was a source of turmoil. Some members, particularly 4F, accused the client of lying and expressed doubts about his morality and honesty. 4F was successful in creating an atmosphere of mistrust and para-

Table 3

Internal Impact of Issue

\begin{tabular}{cl}
\hline Level & \multicolumn{1}{c}{ Internal Impact } \\
\hline 0 & No impact \\
1 & Small changes made to document \\
2 & Large-scale restructuring to one document \\
3 & Major restructuring to more than one document \\
4 & Complete rewrite of documentation \\
5 & No working system at end of project \\
\hline
\end{tabular}


Table 4

External Impact of Issue

\begin{tabular}{cl}
\hline Level & \multicolumn{1}{c}{ External Impact } \\
\hline 0 & Comments and/or concern but no impact \\
1 & Management intervention, leading to small changes \\
2 & Major large-scale restructuring due to management criticism \\
3 & Large-scale restructuring to several documents \\
4 & Marks lost for area of project \\
5 & Deadlines missed, marks lost for late work \\
\hline
\end{tabular}

noia with regard to the client by questioning his sanity and integrity. 4F felt that the client was lying about servers and different technologies that the team could use. This caused other members to question the client's integrity. Some members, particularly $4 \mathrm{~A}$, felt that the client was making statements based on ignorance, but $4 \mathrm{~F}$ argued that he was deliberately lying. $4 \mathrm{~F}$ showed anger and impatience when asking questions. A major problem surfaced when the client said that he was willing to give up the system; this threw the team's plans into chaos for a short period. Another cause for concern was the differing opinions between the team and the client with regard to thirdparty postcode software. The client claimed that thirdparty software was freely available but refused to give any pointers; this caused the team to question whether he was being serious. 4F accused the client of lying about this. The end result was that the system worked but there was no script to set up a postcode. A lot of time was lost as the team concentrated on the postcode issue, as opposed to the more pressing problem of sorting out registration to their system.

This distrust and anger toward the client had a negative impact on the product. The team did not trust the client enough to let him modify any part of the structure of the Database (DB). The client wanted additional DB queries; 4B commented that it would become unwieldy if this was the case. $4 \mathrm{~A}$ also noted that the client's requests were too complex for the existing DB and that they would bring in

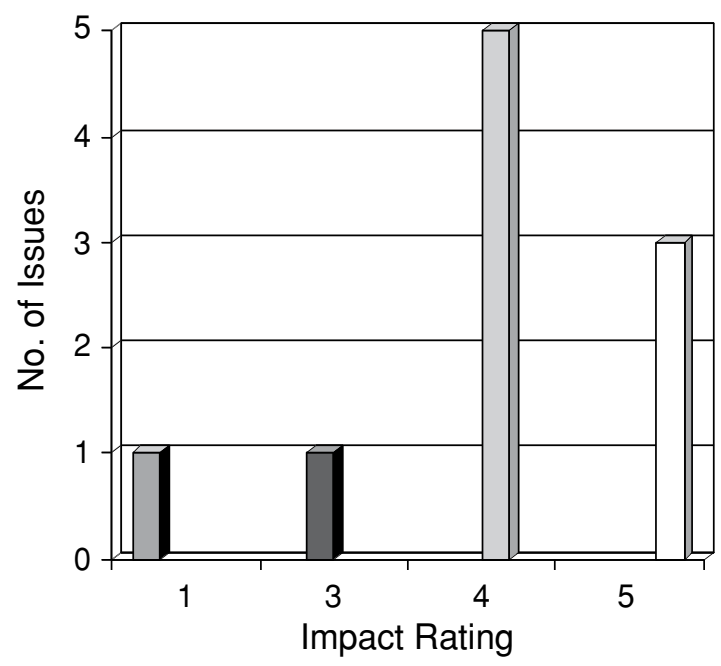

Figure 2. Internal impact for Genesys Team 4. too many new parameters. The client wanted a quick system; 4B said that they would have to trade off quality for time and cost. A lot of categories of the DB were changed; this meant that work was presented that did not have full functionality. The client grew impatient as the team struggled to keep pace with the changing requirements. Minor bugs were uncovered toward the end of the project, but eventually he was happy with what had been done. This situation could have been damaging for the team.

In addition to this, the technical problems were further broken down into people, product, and process categories. The people sector dealt with learning curves - that is, people's getting used to a new concept. This could involve a methodology or simply a lack of experience in largescale team projects. The product category related to issues of a complex technical nature that proved difficult and time consuming for the individuals involved. Finally, the process issues dealt primarily with following a methodology or specific standards, such as the teamwork process in Genesys.

Genesys Team 4 had several problems that were related to the methodology and project protocol. There was a lot of disruptive debate related to project reports. Much of this debate was caused by confusion about the nature and role of documentation in an XP project. This caused anger toward the methodology and confusion about what the team was supposed to be doing with regard to XP. There was confusion about moving from one stage of the project to another. This caused a lot of strain.

Another XP-related problem was that of storycards; there was a lot of disagreement about the use of storycards. This was caused primarily by a lack of experience in XP and anger from 4F. More storycards were needed as the system went on, but the team was not willing to show them to the client; additional comments had to be made to supplement the original storycards, and the team was forced to admit that they were not using storycards correctly. $4 \mathrm{~F}$ argued that taking detailed notes was more effective than using storycards.

This caused panic when the monthly report was due in, since the storycards were not done; this meant that the team had to seek additional advice about storycards, or they would risk losing marks, since marks were allocated by adherence to XP. There were problems with some reports and stories not being updated; marks were docked, and comments were made about this. The XP problems could be put down to a lack of experience on the part of the majority of team members, coupled with some serious personality clashes. This sensing-feeling personality of $4 \mathrm{~B}$, along with that of $4 \mathrm{~A}$, who had experience working with XP, even though he did not adhere to it properly, helped the team to use as many XP practices as possible. The anger from $4 \mathrm{~F}$ tended to stifle a lot of the XP discussion, since he had a strong hostility toward it. Despite these obvious difficulties, thanks to 4B's leadership, the team made good efforts to adhere to such practices as pair programming and collective ownership.

The results show that Genesys Team 4 encountered sociopolitical difficulties. Within these subgroups, different 


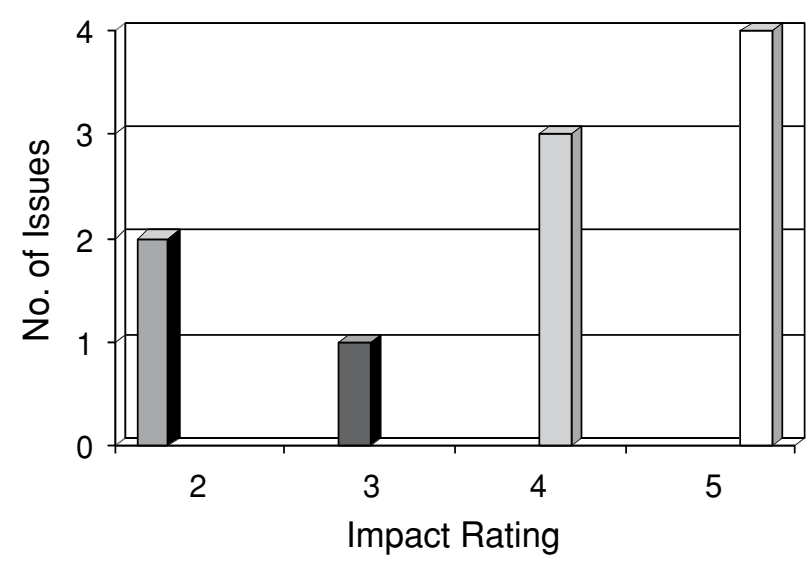

Figure 3. External impact for Genesys Team 4.

patterns emerged, from what one might term personality clashes between members to communication problems, which incorporate a lack of debate.

One such problem was the hostility between $4 \mathrm{~F}$ and $4 \mathrm{C}$, which erupted when $4 \mathrm{C}$ said that he did not want to work for the client. This was part of a deeper problem, which will be explained in more detail below.

The communication problems were the result of a lack of organization at the start of meetings; they had no clearly defined roles and wanted to function as a workers' cooperative. Sometimes, people were appointed to roles against their will; people were indecisive about what they planned to do, and this caused anger from $4 \mathrm{~F}$. The net result of all of this was that work plans were left out of initial monthly reports, resulting in negative managerial feedback, and minutes from previous meetings were not typed up, creating bottlenecks.

The apathy of the team members was exacerbated by a clash between $4 \mathrm{~F}$ and $4 \mathrm{C}$. There was a lack of enthusiasm from certain team members, particularly $4 \mathrm{C}$. This caused an angry reaction from $4 \mathrm{~F}$; as a result of this, efforts to involve 4C were largely futile. 4D and 4C tried to push work onto others and had to be almost forced to participate in meetings. $4 \mathrm{~F}$, by shouting and intimidating, eventually forced $4 \mathrm{C}$ to cut himself off from the project. $4 \mathrm{~B}$ tried to encourage involvement, but the situation had gone too far. $4 \mathrm{~B}$ was forced to remind the team that teamwork was important and Genesys reflected that. 4B said that 4C's apathy was worrying.

This apathy had a detrimental effect on the product. Problems were caused by 4C's not doing the work correctly and not keeping the team informed about their progress; as a result, the same part of the system had to be rewritten four times. 4B had to do a rollback in CVS because of $4 \mathrm{C}$ 's silence. $4 \mathrm{C}$ had to be almost forced to turn up at later meetings, since he had to discuss his part of the project; this looked and was unprofessional.

$4 \mathrm{~F}$ was a key figure in many of the disruptions. 4F's type is ENTJ, with very clear preferences for functions and attitudes. Along with the client, $4 \mathrm{C}$ bore the brunt of
4F's onslaught. The onslaught would have been worse had it not been for the efforts of 4B in particular. 4C's type is INTJ, with very clear preferences. His intuition gave him an iconoclastic imagination and an unhampered view of the possibilities. Routine production would be a waste of his intuition. In this team, $4 \mathrm{C}$ was expected to do certain tasks and was also expected to contribute to meetings. $4 \mathrm{C}$ complained that he found a lot of the project to be boring; therefore, intuition was being restricted and not given full play. Since $4 \mathrm{C}$ was also a natural introvert, it was easy for him to retreat back into his shell when faced with problems, especially with $4 \mathrm{~F}$. By attempting to force $4 \mathrm{C}$ to contribute more, all that was achieved was that $4 \mathrm{C}$ became more distant from the rest of the team and ended up cutting himself off from them. The clear preference for thinking caused problems for $4 \mathrm{C}$ that prevented him from expressing concerns about the personal problems that plagued him for parts of the project. A preference for feeling would have helped $4 \mathrm{C}$ to discuss and be more open about these personal issues. The judging preference allowed $4 \mathrm{C}$ to make a quick decision that he was going to isolate himself and keep contact with the rest of the team to a minimum.

A key factor here was the personality type of the people involved with this team. One member had a boisterous and noisy motivational style, and this alienated him from certain other members of the team, who were quiet, retiring types. Serious disruption was averted by good people management skills; this was impressive when one considers the diversity of this team and the potentially explosive clashes that could have occurred.

\section{Discussion}

Qualitative research offers researchers distinctive opportunities to develop analytic perspectives that speak directly to the practical circumstances and processes of everyday life. Human beings exhibit very complex behavior, and this behavior cannot always be explained by statistics or quantitative data alone. Qualitative research methods allow researchers to delve deeper into the complexities of human behavior. The results from this study show that certain personality types were more predisposed to certain roles and certain kinds of work. Examples of this are the peace-making and excellent people management skills exhibited in Genesys Team 4.

It was also clear that some team members caused more disruption than did others, whether it was through anger, apathy, or incompatibility with another team member and/ or a manager/client. All of these traits were evident; the problems they caused also became self-evident as time progressed.

A very dangerous situation can arise when individuals are reluctant to express opinions about something of concern because no one else has mentioned it or because they lack the assertiveness to put the point forward. Sometimes, people are reluctant to comment because they feel that "Everyone else agrees with the proposed strategy, so I guess it must be all right." This has often been referred to 
as groupthink (Irving, 1989; Janis, 1982) and has been responsible for major disasters, not limited to SE projects.

The actual behavior of the students was measured according to Jungian psychological types; this allowed the authors to ascertain whether given combinations of personalities can work productively over the course of an SE project. It is hoped that this research will provide general pointers to others who seek to carry out ethnographic work in SE.

The results of this work help to explain the conceptual orientation of software engineers toward working through the stages of a project. This study yielded a fascinating insight into team meetings in SE projects and how psychological types can help to support a cohesive team. A whole plethora of issues were raised; a whole plethora of problems arose. Many different personality types were evident, and the discussion encompassed diverse topics from SE techniques to psychological warfare.

\section{Conclusions}

There is no single method that could be said to be the final answer to all of the methodological problems of social research. The use of a method or combination of methods depends on the aim of the research, the practical difficulties that are faced in the field, and the time and money available to conduct the research in the first place. Its success and execution depend on the skills of the researchers and their understanding of the issues that inform research practice. Ethnography was employed successfully as part of a larger project looking into the effects of psychological type on SE teams. The use of this method allowed the authors to gain an insider's understanding of the interactions going on within the student teams. Like any other research method, ethnography also has a set of potential problems that come with it. The subjects under study could be affected, and in particular, this could involve the Hawthorne effect. The Hawthorne effect, along with the possibility of going native, represents two serious problems for someone trying to carry out ethnographyrelated work.

This study did not go entirely according to plan; the process of converting the initial field notes into meaningful data was a particularly long and arduous affair, with many instances of backtracking until the classification scheme became tenable. In some senses, this is to be expected when one realizes how many data were generated in the field notes and that it was the first attempt at carrying out this kind of experimental work in a disciplined research environment. Ethnography is not an easy method to use. It requires researchers to spend a great deal of time in surroundings with which they may not be famil- iar, to secure and maintain relationships with people with whom they may have little personal affinity, to take copious notes on what would normally appear to be everyday happenings, to possibly incur some personal risk in their fieldwork, and, then, to spend months of analysis after the fieldwork.

Ethnography is about engaging in a social scene, experiencing it, and seeking to understand and explain it. The researcher is the medium through which this takes place. By listening and experiencing, impressions are formed, and theories are considered, reflected upon, developed, and modified. It is not an easy method to perform or to analyze, but despite the arguments of its critics, it is a systematic and disciplined study that, if performed well, greatly assists in the understanding of human activities and brings with it new ways of viewing the social world.

\section{REFERENCES}

AtKinson, P. (1990). The ethnographic imagination: Textual constructions of reality. London: Routledge.

BECKer, H. (1993). Problem of inference and proof in participant observation (Reprint ed.). New York: Irvington.

Brooks, F. P. (1975). The mythical man month: Essays on software engineering. Reading, MA: Addison-Wesley.

Costa, P. T., \& McCrae, R. R. (1992). Four ways, five factors are basic. Personality and Individual Differences, 13, 653-665.

Cowling, A. J. (1994). A framework for developing the software engineering curriculum. Proceedings of the ACM/IEEE Sponsored International Workshop on Software Engineering Education (pp. 111-118). Los Alamitos, CA: IEEE Computer Society Press.

Cowling, A. J. (1997). Curriculum support for professionalism. In C. Myers, T. Hall, \& D. Pitt (Eds.), The responsible software engineer: Selected readings in IT professionalism (pp. 285-295). London: Springer.

IRVING, J. (1989). Crucial decisions: Leadership in policymaking and crisis management (pp. 89-117). New York: Free Press.

JANIS, I. L. (1982). Victims of groupthink: A psychological study of foreign policy decisions and fiascoes. Boston: Houghton Mifflin.

Jung, C. G. (1923). Psychological types (Vol. 6). New York: Harcourt.

KARN, J. S., \& Cowling, A. J. (2004a). An initial observational study of the effects of personality type on software engineering teams. In Proceedings of the 8th International Conference on Empirical Assessment in Software Engineering (pp. 155-165). Edison, NJ: IET Press.

Karn, J. S., \& Cowling, A. J. (2004b). An initial study of the effect of personality on group projects in software engineering (Research Rep. CS-04-01). Sheffield, U.K.: University of Sheffield, Department of Computer Science.

KARN, J. S., \& CoWLING, A. J. (2004c). A study into the effect of disruptions on the performance of software engineering teams (Research Rep. CS-04-17). Sheffield, U.K.: University of Sheffield, Department of Computer Science.

Myers, I. B., \& MYers, P. B. (1987). Gifts differing: Understanding personality type. Palo Alto, CA: Davies-Black.

WHYTE, W. F. (1984). Learning from the field: A guide from experience. London: Sage.

(Manuscript received September 22, 2005; revision accepted for publication July 5, 2006.) 